(Manuscript prepared for International Journal of Oral and Maxillofacial Surgery)

\title{
Effect of Bone to Implant Contact Percentage on Bone Remodeling Surrounding a Dental Implant
}

\author{
Zhiqiang Lian ${ }^{\mathrm{a}}$, Hong Guan ${ }^{\mathrm{b}}$, Saso Ivanovski ${ }^{\mathrm{c}}$, Yew-Chaye Loo ${ }^{\mathrm{b}}$, Newell W. Johnson ${ }^{\mathrm{c}}$, Hongwu \\ Zhang $^{\mathrm{a}}$ \\ ${ }^{a}$ State Key Laboratory of Structural Analysis for Industrial Equipment, Dept. of Engineering \\ Mechanics, Dalian University of Technology, Dalian, 116023, China \\ ${ }^{\mathrm{b}}$ Griffith School of Engineering, Griffith University Gold Coast Campus, Queensland 4222, Australia \\ ${ }^{\mathrm{c}}$ School of Dentistry and Oral Health, Griffith University Gold Coast Campus, Queensland 4222, Australia
}

Dec 16, 2008

\author{
Word count of abstract $=190$ words \\ Word count of main text $=4859$ words
}

Address correspondence to Mr. Zhiqiang Lian. State Key Laboratory of Structural Analysis for Industrial Equipment, Dept. of Engineering Mechanics, Dalian University of Technology, Dalian, 116023, China. Telephone number: +86 (0)411 84708408. Fax number: +86 (0)411 84708769. Email: lianzq75@dlut.edu.cn or lianzq75@gmail.com . 


\begin{abstract}
Dental implants are an effective, safe and predictable solution for patients suffering from tooth loss. However implant placement changes the normal mechanical environment of the jawbone leading to bone density redistribution and 'remodeling', in order to to adapt to the new environment. Many bone remodeling theories assume the presence of $100 \%$ contact between bone and implant, which is inconsistent with clinical reality that is about $50 \% \sim 80 \%$ bone-implant contact is commonly seen in clinically successful implant treatment. The influence of different percentages of bone-implant contact on bone remodeling has not been investigated adequately in literature. This study aims to evaluate such an influence using a newly proposed remodeling algorithm through a 2D finite element model. Four different degrees of bone-implant contact $(25,50,75$ and 100\%) are considered and their influences on the density distribution of jawbone is evaluated. The predicted results indicate that no matter what the initial percentages of bone-implant contact (25-100\%) are, the final outcome is about $58 \%-60 \%$ contact when an equilibrium state is reached by bone remodeling. The results are consistent with clinical observations and findings.
\end{abstract}

Keywords: Dental implant, Bone-implant contact, Bone remodeling, Finite element analysis 


\section{Introduction}

A dental implant is a biocompatible titanium device that is surgically placed into the mandibular or maxillary bone to support a prosthetic tooth crown in order to replace missing teeth. Dental implants are an effective, safe and predictable solution for those who have lost a tooth or teeth due to dental caries, periodontal disease, injuries or other reasons. Despite high success rate of implantation when the implants are correctly designed, manufactured and inserted in bone of good quality and quantity, implant failures do exist, especially in compromised sites and patients ${ }^{25}$.

Bone, as a living tissue, is capable of optimizing its internal structure by redistributing its apparent density under the influence of external loads to fulfill its maximum function. This paradigm is known as the Wolff's law ${ }^{38}$. The process of bone redistributing its apparent density is termed as 'bone remodeling'. Since the early publication of Wolff ${ }^{38}$, many theories describing the bone remodeling process have been proposed. Frost ${ }^{12}$ suggested that internal and external remodeling should be distinguished, where internal remodeling refers to the alteration of density of bone tissue and external remodeling is the apposition and removal of bone tissue on the bone surface. Pauwels ${ }^{27}$ concluded that changes in the magnitude of the habitual mechanical stimulation lead to an 'atrophy' or 'hypertrophy' of the osseous structure. Frost ${ }^{12}$ also suggested a trigger criterion for remodeling based on the strain magnitude. This concept was formally developed later by Cowin and his co-workers ${ }^{5,6}$ using the concept of adaptive elasticity under small strains, which was the first mathematically rigorous theory for adaptive growth and remodeling of bone. Cowin and Firoozbakhsh $^{7}$, Firoozbakhsh and Cowin ${ }^{11}$, and Cowin et al. ${ }^{8}$ used the strain tensor as the stimuli of bone remodeling and assumed a quadratic relationship between the strain and the rate of adaptation. Carter $^{3}$ proposed the concept of 'lazy zone', a threshold value, indicating that bone remodeling takes place only when the external stimuli exceed the 'lazy zone'. This concept was incorporated by Huiskes et al. ${ }^{14,15}$ who used the strain energy density as the stimulus signal to control bone remodeling. Mullender et al. ${ }^{22}$ and Mullender and Huiskes ${ }^{23}$ developed a physiological approach to simulate bone remodeling which assumed that osteocytes are sensitive to mechanical loading and are active in controlling the adaptation of bone mass in their environment.

When an implant or implants are surgically placed into the jawbone, the mechanical environment is altered. According to Wolff's law, bone rearranges its internal structure by remodeling in response to implant placement and loading. Although many applications of bone remodeling theories are documented on the prediction of density distribution in peri-implant bone tissues ${ }^{4,13,17,20}$, no 
consideration was taken on the effect of percentage of bone-implant contact on bone remodeling outcomes. A full contact between the bone and implant is often assumed in existing biomechanical research, which assumes that $100 \%$ bone-implant contact is achieved. This is inconsistent with clinical observations, which show that bone-implant contact is dependant on a variety of factors such as implant surface topography and bone quality. The present study, based on a newly proposed bone remodeling algorithm, aims to evaluate the effect of percentage of bone to implant contact on density distribution of jawbone through a two-dimensional finite element model.

\section{Methods}

The bone remodeling theory

Amongst the existing bone remodeling theories ${ }^{14-16,22,23,37,40}$, some popular yet distinctive ones are summarized in the following subsections.

Weinans and Huiskes theory

A popular bone remodeling theory suggested by Huiskes et al. ${ }^{15}$ is expressed as

$$
\frac{d \rho}{d t}=\left\{\begin{array}{ccc}
B\left(\frac{U}{\rho}-(1+s) K\right) & \text { if } & \frac{U}{\rho}>(1+s) K \\
0 & \text { if } & (1-s) K \leq \frac{U}{\rho} \leq(1+s) K \\
B\left(\frac{U}{\rho}-(1-s) K\right) & \text { if } & \frac{U}{\rho}<(1-s) K
\end{array}\right.
$$

where $\rho$ is the apparent density of bone tissue; $d \rho / d t$ is the rate of change in density; $B$ is a constant regulating the rate of the remodeling process; $U$ is the strain energy density (SED); $s$ is in percentage denoting the region of the 'lazy zone' around the threshold value $K$, which is a reference value of SED (Figure 1); and the 'lazy zone' describes the bone not having a net change in apparent density. In Eq. (1), $U$ can be expressed as:

$$
U=\frac{1}{2} \sigma \cdot \varepsilon
$$

where $\sigma$ and $\varepsilon$ are respectively the stress and strain tensors of the bone tissue.

For multiple-load cases, the SED can be expressed as:

$$
U=\frac{1}{n} \sum_{i=1}^{n} U_{i}
$$

where $n$ is the number of load cases and $U_{i}$ is the SED under load case $i$. 
'[Insert Figure 1 about here]'

Similar to Huiskes et al.' theory, Weinans et al. ${ }^{37}$ adopted the strain energy per unit of bone mass, i.e. $U / \rho$ as the bone remodeling stimuli and proposed a time-dependent remodeling equation:

$$
\frac{d \rho}{d t}=B\left(\frac{U_{a}}{\rho}-k\right), 0<\rho \leq \rho_{c b}
$$

where $\rho=\rho(x, y, z)$ is the apparent density of bone tissue at position $(x, y, z) ; U_{a}$ is in the same form as Eq.(3) and $\rho_{c b}$ is the maximum apparent density which is equivalent to that of a cortical bone.

Weinans et al.'s algorithm was applied to predict density distribution in a normal proximal femur with results showing a discontinuous pattern ${ }^{37}$. The algorithm showed an unstable condition in its mathematical operation. Due to the nature of the differential equations used in the algorithm to describe the adaptive remodeling process, the simulation produces discontinuous configurations, a phenomenon called 'checker-board'. As discussed by Ruimerman and Huiskes ${ }^{32}$, the resulting density distributions correlate well with those in a real proximal femur, only when the density distributions were locally averaged from discontinuous density patterns in the femoral head where the trabecular bone is located.

Mullender et al. and Mullender and Huiskes theory

Not having intrinsically different biological nature from the theory of Huiskes et al. ${ }^{15}$ and Weinans et al. ${ }^{37}$, Mullender et al. ${ }^{22}$ and Mullender and Huiskes ${ }^{23}$ suggested a new algorithm which simulated bone remodeling as a self-organizational control process. In their theory, bone is assumed to have $N$ sensor cells distributed uniformly over its volume. An arbitrary sensor $i$ measures a signal $S_{i}$, which is given as:

$$
S_{i}=\frac{U_{i}}{\rho_{i}}
$$

where $U_{i}$ is the SED and $\rho_{i}$ is the apparent density at the location of the sensor. The density $\rho(\mathbf{x}, t)$ at location $\mathbf{x}$ is regulated by the stimulus value $\Phi(\mathbf{x}, t)$, to which all sensor cells contribute, relative to their distance from $\mathbf{x}$. Hence,

$$
\Phi(\mathbf{x}, t)=\sum_{i=1}^{N} f_{i}(\mathbf{x})\left(S_{i}-K\right)
$$

where $N$ is the total number of sensors; $K$ is a reference signal of SED and $f_{i}(\mathbf{x})$ is a spatial influence function given as: 


$$
f_{i}(\mathbf{x})=e^{-\left[d_{i}(\mathbf{x}) / D\right]}
$$

where $d_{i}(\mathbf{x})$ is the distance between sensor $i$ and location $\mathbf{x} ; D$ is the rate of the spatial influence reduction. Note that $D$ represents the distance from a sensor at which location its effect is reduced to $e^{-1}$, i.e. $36.8 \%$. This is illustrated in Figure 2.

'[Insert Figure 2 about here]'

The density $\rho(\mathbf{x}, t)$ is now governed by:

$$
\frac{d \rho(\mathbf{x}, t)}{d t}=\tau \Phi(\mathbf{x}, t), \text { with } 0<\rho(x) \leq \rho_{\max }
$$

where $\rho_{\max }$ is the maximum density equivalent to that of a cortical bone, and $\tau$ is a time constant regulating the rate of the remodeling process.

This algorithm was used to predict the density distribution of a 2D plate model previously studied by Mullender et al. ${ }^{22}$. The model produced trabecular-like structures without the 'checker-board' patterns. It should be noted that the checker-board problem produced by the phenomenological models like the one of Weinans et al. ${ }^{37}$ has been completely solved by different techniques including non-local stimuli ${ }^{22,23}$ or using extrapolation method to the nodal points ${ }^{16}$. The checker-board phenomenon is believed to be attributable to the softening induced by resorption rather than remodeling itself. When using the non-local definition of the stimulus as a sum of the signal of different osteocytes near the point concerned, Mullender et al.'s model ${ }^{22,23}$ does not produce 'checker-board' patterns.

Based on the theories of Weinans et al. ${ }^{37}$ and Mullender et al. ${ }^{22}$, Zhu et al. ${ }^{40}$ proposed a new bone remodeling theory which includes a high-order non-linear effect. The governing equation is:

$$
\begin{gathered}
\frac{d \rho(x, t)}{d t}=B\left(\sum_{i=1}^{N} f_{i}(x)\left(\beta_{i}^{\alpha}-1\right), \text { with } 0<\rho(x) \leq \rho_{c b}\right. \\
\beta_{i}=\frac{U_{a}(i)}{\rho_{i} K}
\end{gathered}
$$

where $\beta$ is a comparative coefficient describing the comparison of mechanical stimulus $U_{a}(i) / \rho_{i}$ in each sensor cell with reference value $K$; $\alpha$ is the order of non-linear remodeling equation.

Zhu et al.'s remodeling algorithm is able to control the remodeling process more effectively due to 
the consideration of non-linear effect. However, similar to Mullender's theory, the 'lzay zone' effect was not included in their equations.

\section{The proposed algorithm}

The abovementioned two types of theories have both shown to exhibit advantages and disadvantages. Huiskes's theory takes into account the 'lazy zone' effect but is unstable in its mathematical operation leading to a discontinuous and an inevitable 'checker board' pattern. Mullender and Zhu et al.'s theories, on the other hand, are physiologically and mechanically more consistent and the 'checker board' phenomena can be effectively eliminated. However the effect of 'lazy zone' was not considered. The 'lazy zone' effect, initially proposed by $\mathrm{Carter}^{3}$ based on experimental investigation, has been verified by Rubin and Lanyon ${ }^{31}$ to be an important factor which should be considered in the simulation of the remodeling process. To overcome the shortcomings of the above theories, a new algorithm combing these theories is proposed herein.

In the proposed algorithm, the bone is assumed to have $N$ sensor cells distributed uniformly over its volume ${ }^{22}$. An arbitrary sensor $i$ measures a signal $S_{i}$, which is given as:

$$
S_{i}=\frac{U_{i}}{\rho_{i}}
$$

where $U_{i}$ is the strain energy density; and $\rho_{i}$ is the density at the location of the sensor. For multiple-load cases, the strain energy density can be expressed as:

$$
U=\frac{1}{n} \sum_{i=1}^{n} U_{i}
$$

where $n$ is the number of load cases and $U_{i}$ is the strain energy density under load case $i$.

The density $\rho(\mathbf{x}, t)$ at the location $\mathbf{x}$ is regulated by the stimulus value $\Phi(\mathbf{x}, t)$, to which all sensor cells contribute, relative to their distance from $\mathbf{x}$. Hence,

$$
\Phi_{i}(x, t)=\left\{\begin{array}{clc}
\sum_{i}^{N} f_{i}(x)\left(S_{i}^{\alpha}-(1+s) K\right) & \text { if } & S_{i}>(1+s) K \\
0 & \text { if } & (1-s) K \leq S_{i} \leq(1+s) K \\
\sum_{i}^{N} f_{i}(x)\left(S_{i}^{\alpha}-(1-s) K\right) & \text { if } & S_{i}<(1-s) K
\end{array}\right.
$$

where $N$ is the total number of sensors; $\alpha$ is the order of non-linear remodeling equation; $K$ is a reference signal of strain energy density per unit bone mass; $s$ is in percentage denoting the region of the 'lazy zone' around the threshold value $K$. The density $\rho(\mathrm{x}, t)$ is governed by the remodeling rate as expressed in Eq. (8) and $f_{i}(\mathbf{x})$ is a spatial influence function as given in Eq. (7). 
The applicability of the proposed algorithm on density distribution of bone has been verified using Mullender et al's 2D plate model as well as a simplified implant model ${ }^{18}$.

\section{The finite element procedure}

As the apparent density of bone tissue changes during the process of remodeling, Young's modulus of bone changes accordingly. Some empirical relationships between the apparent density and the mechanical properties of bone have been established experimentally ${ }^{2,9,29}$. Young's modulus $E$ of bone tissue at location $\mathbf{x}$ is calculated according to Currey ${ }^{9}$ and Rice et al. ${ }^{29}$ as

$$
E(\mathbf{x}, t)=C \rho(\mathbf{x}, t)^{\gamma}
$$

where $\rho$ is the apparent density of bone tissue; $C$ and $\gamma$ are the constants which can be determined through experiment ${ }^{2,9,29}$.

In the iterative finite element analysis process, the governing equation (Eq. (8)) is necessary to be re-written as:

$$
\rho_{i}(t+\Delta t)=\rho_{i}(t)+\Delta t \tau \Phi_{i}(t)
$$

where $\Delta t$ is the time step in the iteration process and $\Phi_{i}(t)$ is in the form of Eq. (13). The iteration is continued until no more significant changes take place in density distribution.

The remodeling procedure is outlined as follows:

(1) Create a finite element model of implant-bone system. Apply load and boundary conditions. Assign uniformly distributed initial values of Young's modulus and density to the bone.

(2) Perform a linear static finite element analysis.

(3) For each element, a sensor is located at the centre of the element. For an arbitrary sensor $i$, calculate the stimulus $\Phi_{i}(\mathbf{x}, t)$ based on Eq. (13) and the density value based on Eq. (15). Such calculation is done for all sensors (elements) and some of which may have their original densities changed to new values.

(4) Is a density convergence reached? if 'YES', the procedure of remodeling is terminated. If 'NOT', then continue to the next step.

(5) Update the values of Young's modulus based on Eq. (14) which in turn updates the finite element model.

(6) Go to step 2. 


\section{Finite Element Analysis Simulation}

To evaluate the effect of different bone-implant contact on the density distribution of jawbone surrounding a dental implant, a 2D slice at the position of first premolar is taken from the entire mandibular bone. The system of crown, abutment, abutment screw, implant and bone are modeled using 2D plane strain elements. Two different types of bone, i.e. cortical and cancellous bone, are identified in the model based on CT scan images. The implant dimensions are based on those of $\mathrm{Neoss}^{24}$. Fig. 3(a) shows an implant of diameter $4.5 \mathrm{~mm}$ and length $11 \mathrm{~mm}$, and a mandible section with a cortical bone thickness of $1 \mathrm{~mm}$. The implant is conical with 2 degrees of taper and has a helical thread. A small area of bone surrounding the implant $(0.2 \mathrm{~mm}$ from tip of implant thread $)$ is designated as connective tissue, constituting a mix of hard and soft tissue to simulate varying degrees of bone to implant contact. This is also detailed in Fig. 3(a). In this particular finite element model, the total numbers of elements are respectively 759 for the crown, 147 for the abutment, 312 for the abutment screw, 1335 for the implant, 1274 for the cortical bone, 2897 for the cancellous bone, and 408 for the connective tissue. Based on the work of Chou et al. ${ }^{4}$, the implant system is loaded with an occlusal load of $100 \mathrm{~N}$ on the crown at an angle of $11^{\circ}$ and a uniformly distributed pressure of $500 \mathrm{kPa}$ on the outer surface of the cortical bone. The pressure load is to more realistically simulate the boundary conditions. The load and boundary conditions are detailed in Fig. $3(b)$.

'[Insert Figure 3 about here]'

It is commonly known that bone is an orthotropic or anisotropic material. However, in most finite element simulation of bone tissues, an isotropic material behavior of bone is often assumed. This is mainly due to the following considerations: (1) It is difficult to determine the material orientation of bone; (2) Peng et al. ${ }^{28}$ conducted a finite element comparative study on a femur with two different material properties (i.e. isotropic and orthotropic materials). Little difference was found in the results due to the two different material assumptions; (3) the relationships between Young's modulus and apparent density of bone are mostly established based on the isotropic assumption. For the above reasons, an isotropic material behavior is also assumed in the present study.

An extensive literature review by van Staden et al. ${ }^{35}$ indicates that the assumed range of Young's modulus for the cortical bone varies from 5.57 to 22.8GPa and that for the cancellous bone varies from 0.08 to $7.93 \mathrm{GPa}$. In the present study, typical values of Young's modulus $(E)$ of bone, implant components as well as crown are selected based on the work of Menicucci et al. ${ }^{21}$. They are also 
indicated in Fig. 3(b). The Poisson's ratio for all components is taken as 0.3.

When an implant is surgically placed into jawbone, the interface between bone and implant can be divided into two regions, i.e. regions with direct bone-implant contact and those without direct contact $^{33}$. To simulate different degrees of bone-implant contact, a certain percentage of connective tissue elements are randomly selected and assigned the properties of bony tissue. The remaining elements within the connective tissue are assigned the properties of soft tissue (Fig. 4). According to Lu et al. ${ }^{19}$ and Xing et al. ${ }^{39}$, Young's moduli of the bony and soft are taken as 2.4GPa and 70MPa, respectively. In this study, four different degrees of bone-implant contact (i.e. 25, 50, 75 and 100\%) are considered to evaluate their effect on the density distribution of jawbone. Note that $25 \%$ bone-implant contact represents $25 \%$ bony tissue and $75 \%$ soft tissue.

\section{'[Insert Figure 4 about here]'}

In the remodelling process, the density distribution of cortical bone is assumed to be unchanged. However the ratio of bony-soft tissue keeps changing during the process. Hence the connective tissue is also remodelled together with cancellous bone to represent a clinical reality. The cancellous bone has a uniform initial density distribution of $\rho=1.279 \mathrm{~g} / \mathrm{cm}^{3}$. This corresponds to a Young's modulus of $7.93 \mathrm{GPa}$, which is calculated by a specific relationship between the elastic modulus and density as suggested by Carter and Hayes ${ }^{2}$, i.e:

$$
E(x, t)=3790 \times \rho^{3}
$$

where $E$ is Young's modulus; $\mathbf{x}$ is the location of sensors; $t$ is the remodeling time and $\rho$ is the apparent density of bone tissue.

It is worthwhile noting that there are no standard rules in the determination of bone remodeling parameters as specified in Eqs. (13) and (15). Such parameters as $K$, the reference signal of SED; $s$, the 'lazy zone' effect and $\tau$, the time constant regulating the remodeling rate are found to be quite different in the literature. They are enumerated in Table 1.

'[Insert Table 1 about here]'

In order to compare the effect of four different bone-implant contact, the following bone remodeling parameters are kept constant for all four scenarios. According to Chou et al. ${ }^{4}$, the maximum and 
minimum densities are assumed to be $\rho_{\max }=1.5347 \mathrm{~g} / \mathrm{cm}^{3}$ and $\rho_{\min }=0.064 \mathrm{~g} / \mathrm{cm}^{3}$ respectively which corresponds to Young's modulus of $13.7 \mathrm{GPa}$ and $1 \mathrm{kPa}$ respectively; the sensor cell distribution is assumed to be uniform and is located at the centre of each element; the influence reduction parameter $D=0.2 \mathrm{~mm}$. Using Table 1 as a guideline, other parameters are chosen as: $s=20 \%, \tau=1$ $\left(\mathrm{g} / \mathrm{cm}^{3}\right)^{2} /(\mathrm{MPa}$ time-unit) and $K=16 \mathrm{Nm} / \mathrm{kg}$.

\section{Results and Discussion}

To evaluate the effect of different bone-implant contact on the density distribution of jawbone surrounding a dental implant, a 2D representation of the implant and mandible is studied. This is a preliminary study dealing with a new bone remodeling algorithm aimed at overcoming the limitations of the existing methodologies. For the purpose of initial verification, a 2D analysis is undertaken to obtain fundamental understanding of the remodeling process. A more sophisticated 3D analysis will be conducted in the future so that detailed implant geometry can be considered and more accurate solutions be obtained.

Fig. 5 presents the outcome of a progressive remodeling process for a $50 \%$ bone-implant contact scenario. The density distributions in the cancellous bone and connective tissue vary from the original to the converged stages, corresponding to iterations 0, 50, 200, 300, 800 as shown in Figs. 5(a) to (e)). As evident in Fig. 5 that large areas of bone resorption and surrounding formation take place directly under the implant at early iteration (around $50^{\text {th }}$ ) stages. This morphology remains almost unchanged throughout the remaining remodeling process. At approximately $200^{\text {th }}$ iteration, further resorption and formation occur at around both sides of the implant. Again this morphology changes very little until the converged stage. Between iterations 200 and 800, the overall changes in the density distribution are minor. However the total area of bone resorption increseas.

'[Insert Figure 5 about here]'

The predicted results of density distributions in the cancellous bone and connective tissue for four different bone-implant contact scenarios are shown in Fig. 6. As can be seen the final density distributions are almost identical for four different bone-implant contact percentages. The only noticeable difference is the area located at the bottom right side of the implant where the bone tissue demonstrates an average density. This is particularly true for $50 \%-100 \%$ bone-implant contact 
scenarios. For the $25 \%$ contact, this area is a mix between bone resorption and formation.

'[Insert Figure 6 about here]'

Fig. 7 presents a comparison of the predicted density distribution results with clinical observations. Shown in Fig. 7(a) is the predicted result due to the present study for $50 \%$ bone-implant contact scenario. Fig. 7(b) is the prediction of Chou et al. ${ }^{4}$ and Fig. 7(c) displays the density distribution on a baboon's jawbone surrounding a dental implant ${ }^{36}$. The overall density distributions in the three figures are quite similar in the following aspects: (1) the trabecular-like pattern surrounding the implant as highlighted by region A; (2) the lower density area (bone resorption) below the implant as highlighted by region $\mathrm{B}$.

It should be noted that Chou et al.'s ${ }^{4}$ algorithm is based on Huiskes et al.'s ${ }^{15}$ theory with a consideration of high-order effect. However, the 'lazy-zone' effect was ignored. In addition, with a focus on the influence of different types of implant systems on the density distribution, their analysis did not cover varying bone-implant contact percentages.

\section{'[Insert Figure 7 about here]'}

Shown in Fig. 8 is the relationship between bone-implant contact percentage and bone mass after remodeling is complete. As can be seen, the bone mass reaches its maximum value for $100 \%$ contact and a similar bone mass is achieved for $50 \%$ and $75 \%$ contact. Furthermore, the difference between the maximum ( $100 \%$ contact) and minimum (25\% contact) bone mass is less than $0.5 \%$. This suggests that the assumed contact percentage has little influence on the bone mass upon completion of bone remodeling.

Fig. 9 further compares the bone-implant contact percentage before and after bone remodeling. Again initially assumed different percentages result in a rather constant (between 58-60\%) percentage of contact after bone remodeling.

Figs. 6, 8 and 9 all demonstrate that the initial bone-implant percentages have little impact on the final density distribution and bone mass. Upon reaching a balanced bone resorption and formation throughout the process of bone remodeling, the bone-implant contact percentages remain almost constant at about $59 \%$. Testiri et al. ${ }^{34}$ found a bone-implant contact percentage of $64.2 \%$ for an 
immediately loaded implant. Through a histology study, Degide et al. ${ }^{10}$ also discovered a contact percentage of about $65 \%$ to $70 \%$ for two immediately loaded implants. This further verifies that the findings of the present study are in a good agreement with those of the published literature.

$$
\text { '[Insert Figure } 8 \text { about here]' [IInsert Figure } 9 \text { about here]' }
$$

Other similar investigations on bone-implant contact include that of Papavasiliou et al. ${ }^{26}$ who pointed out that an implant never achieves $100 \%$ contact with surrounding bone. Roberts ${ }^{30}$ also stated that even 'clinically successful' implants have less than half of direct contact with bone. In addition, Block et al. ${ }^{1}$ found similar levels of bone-implant contact in dogs. All these investigations suggested a range of $50 \%$ to $80 \%$ bone-implant contact in 'clinically successful' implants, which are consistent with our predicted results.

In this study, the influence of different degrees of bone-implant contact on bone remodeling following dental implantation is evaluated using a newly proposed remodeling algorithm. A two-dimensional finite element model is established and four representative percentages of bone-implant contact are considered in the bone remodeling simulation. The study demonstrates that the initial percentages of bone-implant contact (25-100\%) have little influence on the final density distribution in the bone. Further, a final contact percentage of about $58 \%-60 \%$ is resulted when an equilibrium state is reached by bone remodeling. The results are consistent with the clinical observations and findings.

\section{Declarations}

Funding: This work was funded by the National Science Nature Foundation (No. 10402007, 14021202) and 2006 Griffith University Research Grant scheme

Competing Interests: We, the authors of the manuscript, declare that we have no disclose any financial and personal relationships with other people or organizations that could inappropriately influence (bias) their work.

Ethical Approval: Not required 


\section{References}

1. Block MS, Finger IM, Fontenot MG, Kent JN. Loaded hydroxylapatite-coated and grit-blasted titanium implants in dogs. Int J Oral Maxillofac Implants 1989: 4: 219-225.

2. Carter DR, Hayes WC. The compressive behavior of bone as a two-phase porous structure. J Bone Joint Surg Am 1977: 59: 954-962.

3. Carter DR. Mechanical loading histories and cortical bone remodeling. Calcif Tissue Int 1984; 36: S19-S24.

4. Chou HY, Jagodnik JJ, Muftu S. Predictions of bone remodelling around dental implant systems. J Biomech 2008: 41, 1365-1373.

5. Cowin SC, Hegedus D. Bone remodelling I: theory of adaptive elasticity. J Elastcity 1976: 6: 313-326.

6. Cowin SC, Nachlinger RR. Bone remodelling III: uniqueness and stability in adaptive elasticity theory. $\mathbf{J}$ Elasticity 1978: 8: 285-295.

7. Cowin SC, Firoozbakhsh K. Bone remodelling of diaphysial surfaces under constant load: theoretical predictions. J Biomech 1981: 14: 471-484.

8. Cowin SC, Hart RT, Baber JR, Kohn DH. Functional adaptation in long bones: establishing in vivo values for surface remodelling rate coefficients. J Biomech 1985: 18: 665-684.

9. Currey JD. The effect of porosity and mineral content on the Young's modulus of elasticity of compact bone. J Biomech 1988: 21: 131-139.

10. Degide M, Petrone G, Lezzi G, Piattelli A. Histologic evaluation of 2 human immediately loaded and 1 titanium implants inserted in the posterior mandible and submerged retrieved after 6 months. J Oral Implantol 2003: 29(5):223-229.

11. Firoozbakhsh K, Cowin SC. An analytical model of Pauwels' functional adaptation mechanism in bone. J Biomech Eng 1981: 103: 246-252.

12. Frost HM. The laws of bone structure. Springfield: Thomas C C, 1964.

13. Grupi V, Guglielmino E, La Rosa G, Vander Sloten J, Van Oosterwyck H. Numerical analysis of bone adaptation around an oral implant due to overload stress. Proc Inst Mech Eng [H] 2004: 218: 407-415.

14. Huiskes R, Weinans H, Grootenboer HJ, Dalstra M, Fudala B, Slooff TJ. Adaptive bone-remodeling theory applied to prosthetic design analysis. J Biomech 1987: 20: 1135-1150.

15. Huiskes $R$, Weinans $H$, van Rietbergen $B$. The relationship between stress shielding and bone resorption around total hip stems and the effects of flexible materials. Clin Orthop Relat Res 1992: 274: 124-134.

16. Jacobs CR, Simo JC, Bearpre GS, Carter DR. Adaptive bone remodeling incorporating simultaneous density and anisotropy considerations. J Biomech 1997: 30: 603-613.

17. Li JY, Li HY, Shi L, Foa AS, Ucer C, Devlin H, Horner K, Silkas N. A mathematical model for simulating the bone remodeling process under mechanical stimulus. Dent Mater 2007: 23: 1073-1078.

18. Lian ZQ, Guan H, Ivanovski S, Loo YC, Johnson NW. Bone remodeling simulation in human jawbone surrounding dental implant. Int J Oral Maxillofac Implants 2008(submitted)

19. Lu J, Pan KF, Xu XL, Qu HT. Influence of different bone contact ratio to stress distribution in bone around dental implant (in Chinese). J Oral Maxillofac Surg 2005: 15: 234-237.

20. Mellal A, Wiskott HWA, Scherrer SS, Belser UC. Stimulating effect of implant loading on surrounding bone. 
Comparison of three numerical models and validation by in vivo data. Clin Oral Implants Res 2004: 15: 239-248.

21. Menicucci G, Mossolov A, Mozzati M, Lorenzetti M, Preti G. Tooth-implant connection: some biomechanical aspects based on finite element analyses. Clin Oral Implants Res 2002: 13: 334-341.

22. Mullender MG, Huiskes R, Weinans H. A physiological approach to simulation of bone remodelling as a self-organizational control process. J Biomech 1994: 27: 1389-1394.

23. Mullender M G, Huiskes R. Proposal for the regulatory mechanism of Wolff's law. J Orthop Res 1995: 13: 503-512.

24. Neoss Limited. Neoss Implant System Surgical Guidelines, UK, 2006.

25. Neukam FW, Flemmig TF, Working Group 3. Local and systemic conditions potentially compromising osseointegration. Consensus report of Working Group 3. Clin Oral Implants Res. 2006 :17:160-162.

26. Papavasiliou G, Kamposiora P, Bayne SC, Felton DA. 3D-FEA of bone-implant contact percentages and patterns on implant-bone interfacial stresses. J Dent 1997: 25: 485-491.

27. Pauwels F. Biomechanics of the locomotor apparatus. Berlin: Springer, 1965.

28. Peng L, Bai J, Zeng X, Zhou Y. Comparison of isotropic and orthotropic material property assignments on femoral finite element models under two loading conditions. Med Eng Phys 2006: 28: 227-233.

29. Rice JC, Cowin SC, Bowman JA. On the dependence of the elasticity and strength of cancellous bone on apparent density. J Biomech 1988: 21: 155-168.

30. Roberts WE. Bone tissue interface. J Dent Educ 1988: 52: 804-809.

31. Rubin CT, Lanyon LE. Regulation of bone mass by mechanical strain magnitude. Calcif Tissue Int 1985: 37: 411-417.

32. Ruimerman R, Huiskes R. Development of a unifying theory for mechanical adaptation and maintenance of trabecular bone. Theor Issues Ergon Sci 2005: 6: 225-238

33. Simmons CA, Meguid SA, Pilliar RM. Mechanical regulation of localized and appositional bone formation around bone-interfacing implants. J Biomed Mater Res 2001: 55: 63-71.

34. Testori T, Szmukler-Moncler S, Francetti L, Del Fabbro M, Trisi P, Weinstein RL. Healing of Osseotite implants under submerged and immediate loading conditions in a single patient: a case report and interface analysis after 2 months. Int J Periodontics Restorative Dent 2002: 22: 345-353.

35. van Staden RC, Guan H, Loo YC. Application of the finite element method in dental implant research. Comput Methods Biomech Biomed Engin 2006: 9: 257-270.

36. Watzak G, Zechner W, Ulm C, Tangl S, Tepper G, Watzek G. Histologic and histomorphometric analysis of three types of dental implants following 18 months of occlusal loading: a prelimary study in baboons. Clin Oral Implants Res 2005: 16: 408-416.

37. Weinans H, Huiskes R, Grootenboer HJ. The behavior of adaptive bone-remodeling simulation models. J Biomech 1992: 25: 1425-1441.

38. Wolff JL. The law of bone remodelling. Translated by Maquet P, Furlong R, in 1986. Berlin: Springer-Verlag, 1892.

39. Xing XJ, Liu BL, Liu L. The influence of osseointegration percentage on implant-bone interfacial stresses (in 
Chinese). J Xi'an Jiaotong Univ (Med Sci) 2002: 23: 395-397.

40. Zhu XH, Gong H, Zhu D, Gao BZ. A study of the effect of non-linearities in the equation of bone remodelling. J Biomech 2002: 35: 951-960. 


\section{Figure Legends}

Figure 1. The assumed local adaptation as a function of the SED with the effect of 'lazy zone'.

Figure 2. Relationship between $f(\mathbf{x})$ and $d(\mathbf{x})$.

Figure 3. Finite element model of implant and jawbone: (a) Dimensions; (b) Load, boundary conditions and material properties.

Figure 4. Illustration of different bone-implant contact in connective tissue.

Figure 5. Progressive alteration of density distribution in mandible (50\% bone-implant contact).

Figure 6. Density distribution in cancellous bone and connective tissue under different bone-implant contact.

Figure 7. Comparison between predicted results and clinical observations. (a) Predicted result with 75\% bone-implant contact; (b) Predicted result by Chou et al. ${ }^{4}$; (c) Density distribution in baboons' jaw $^{36}$

Figure 8. Relationship between bone-implant contact percentage and bone mass after bone remodeling.

Figure 9. Comparison of bone-implant contact percentage before and after bone remodeling.

Table 1. Bone remodelling parameters used in published literature 


\section{Figures}

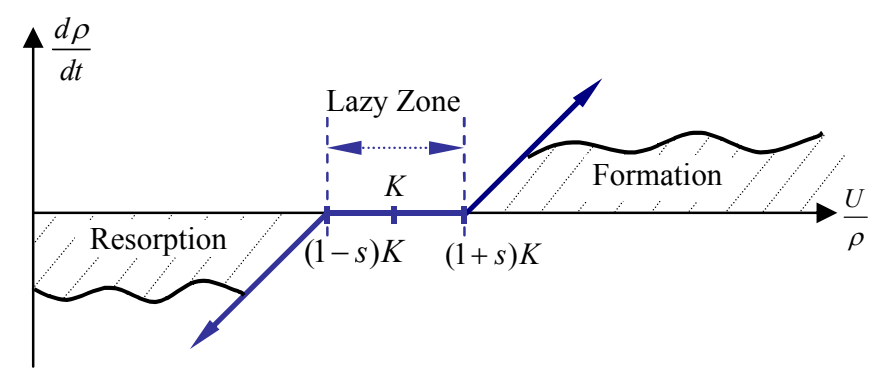

Fig. 1. The assumed local adaptation as a function of the SED with the effect of 'lazy zone'.

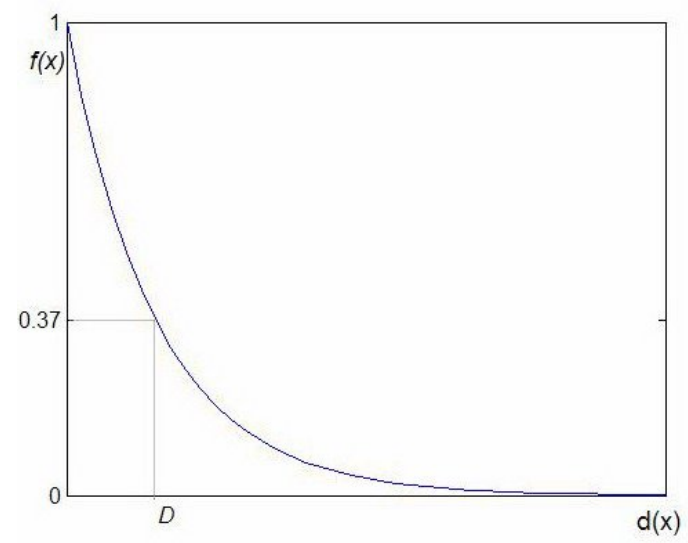

Fig. 2. Relationship between $f(\mathbf{x})$ and $d(\mathbf{x})$. 

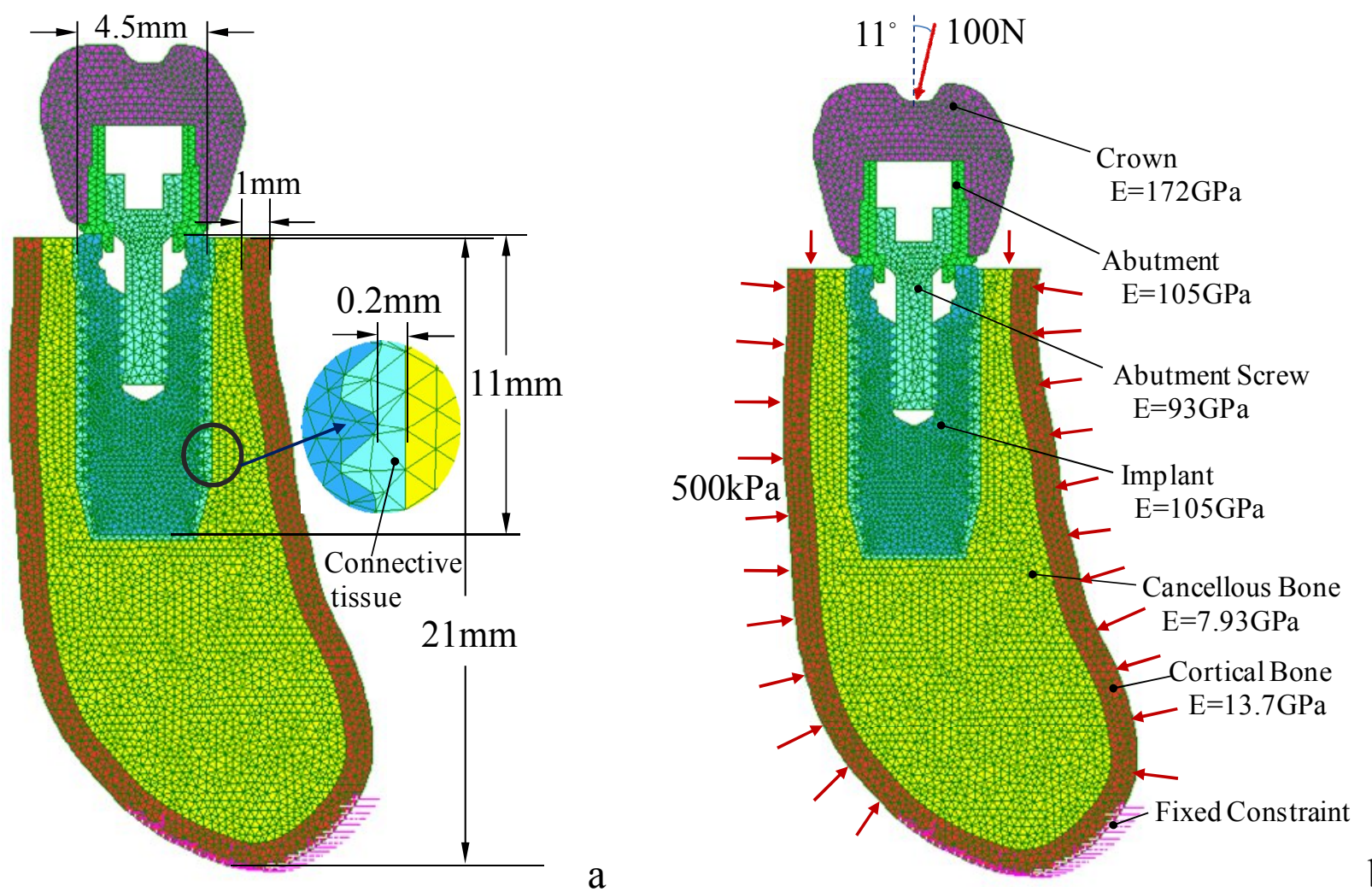

Fig. 3. Finite element model of implant and jawbone: (a) Dimensions; (b) Load, boundary conditions and material properties.

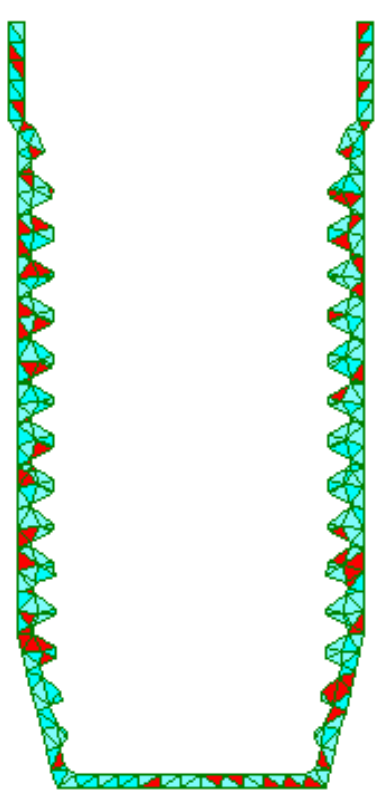

(a) $25 \%$

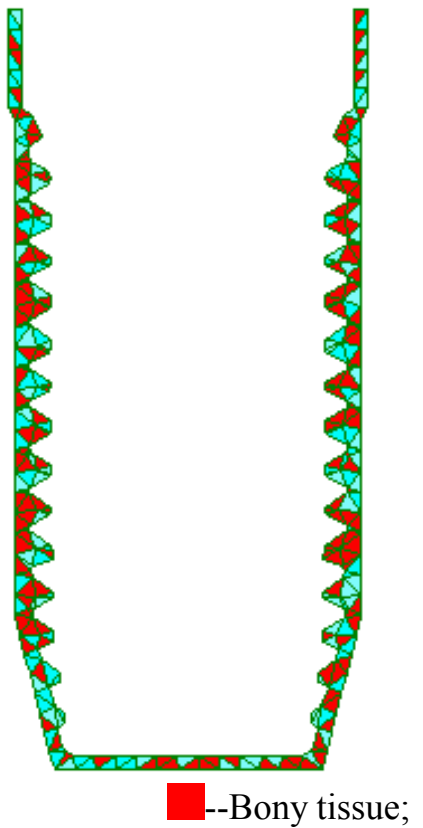

(b) $50 \%$

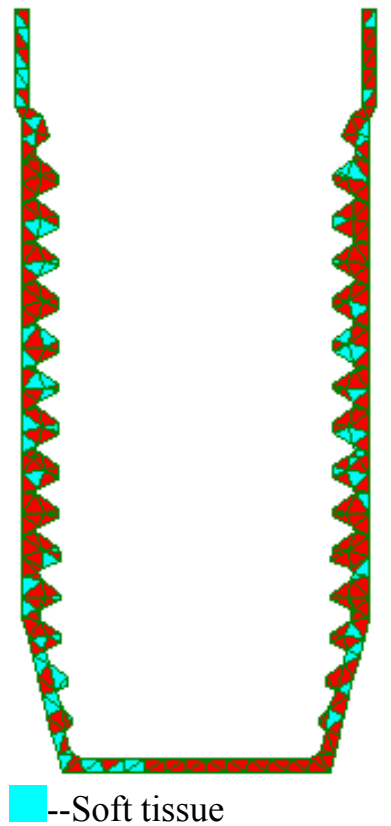

(c) $75 \%$

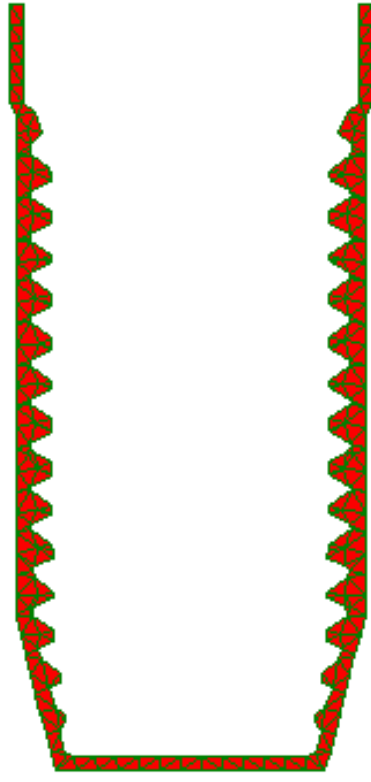

(d) $100 \%$

Fig. 4. Illustration of different bone-implant contact in connective tissue. 


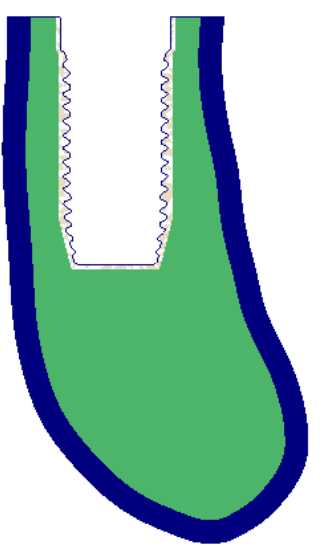

$0.064 \mathrm{~g} / \mathrm{cm}^{3}$

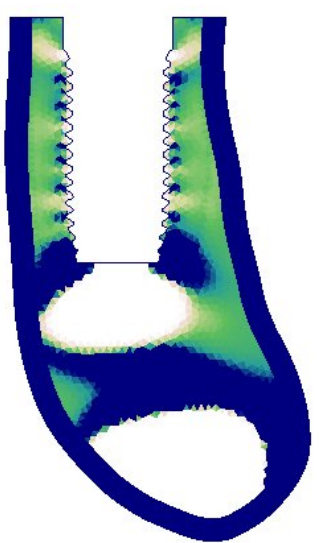

(b) Iteration 50

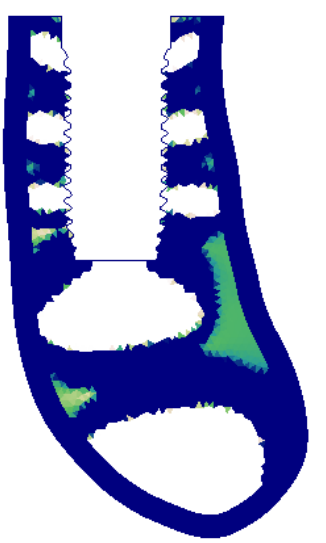

(c) Iteration 200

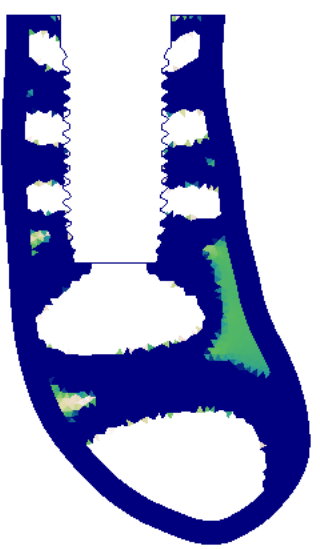

$1.5347 \mathrm{~g} / \mathrm{cm}^{3}$

(d) Iteration 300

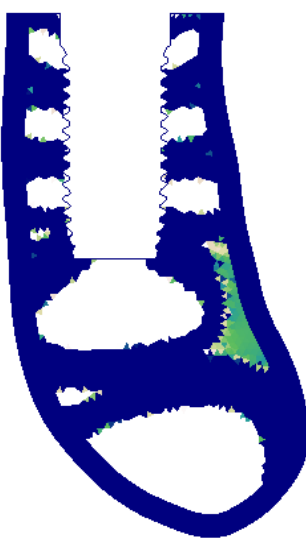

(e) Iteration 800

Fig. 5 Progressive alteration of density distribution in mandible (50\% bone-implant contact) 


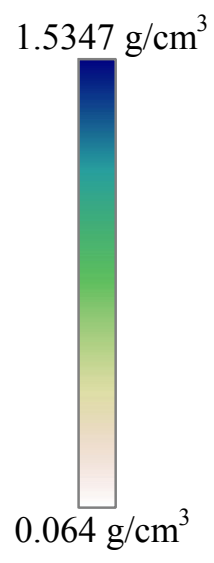

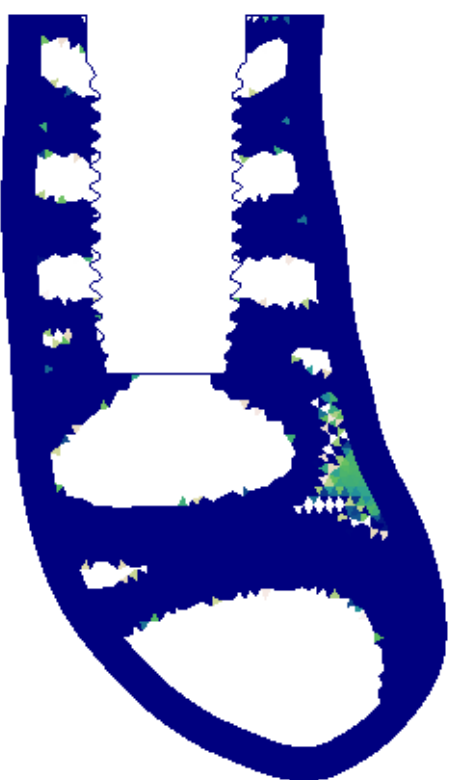

(a) $25 \%$

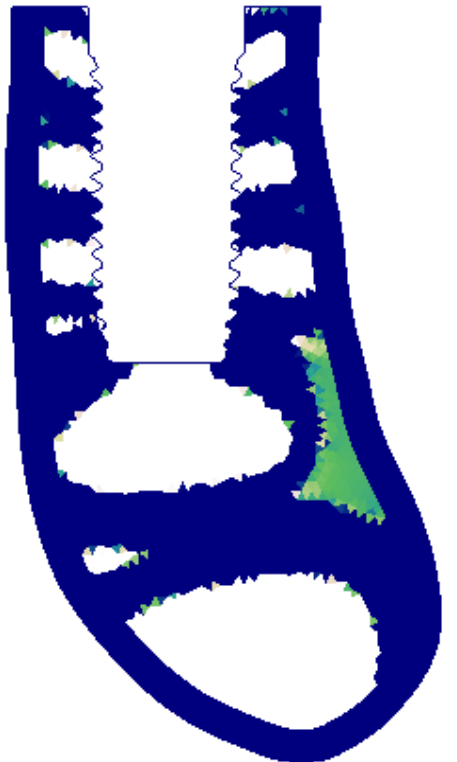

(c) $75 \%$

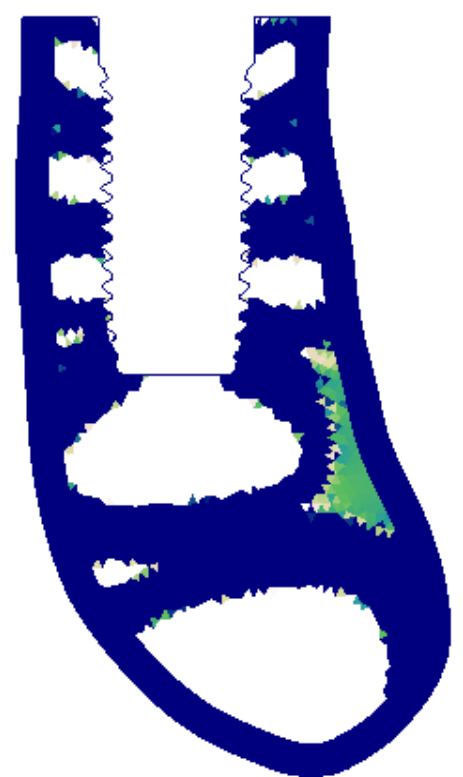

(b) $50 \%$

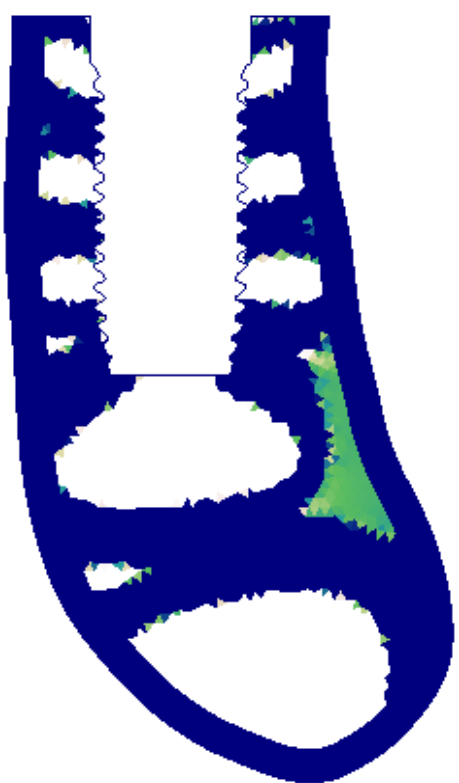

(d) $100 \%$

Fig. 6. Density distribution in cancellous bone and connective tissue under different bone-implant contact 

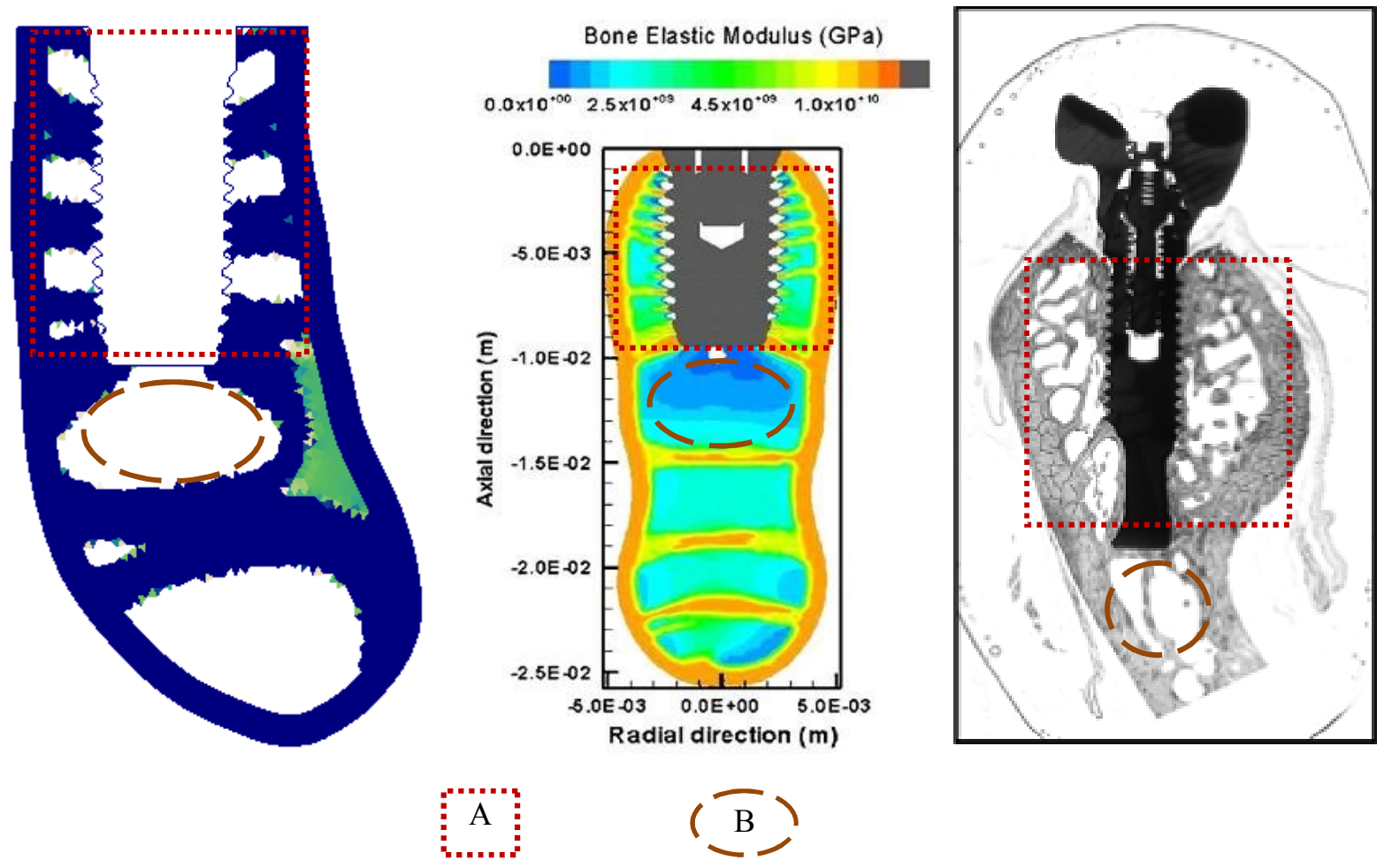

(a)

(b)

(c)

Fig. 7. Comparison between predicted results and clinical observations. (a) Predicted result with $75 \%$ bone-implant contact; (b) Predicted result by Chou et al. ${ }^{4}$; (c) Density distribution in baboons' jaw $^{36}$

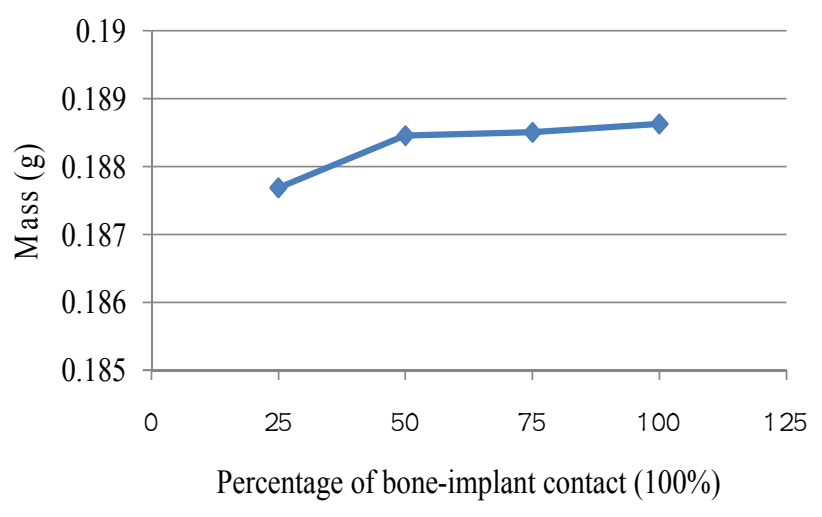

Fig. 8 Relationship between bone-implant contact percentage and bone mass after bone remodeling.

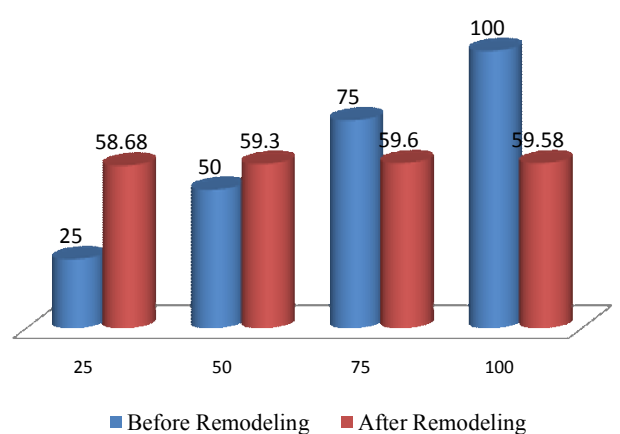

Fig. 9 Comparison of bone-implant contact percentage before and after bone remodeling. 


\section{Tables}

Table 1. Bone remodelling parameters used in published literature

\begin{tabular}{lccc}
\hline & $K$ & $s$ & $\tau$ or $\tau \Delta t$ \\
\hline Chou et al. $^{4}$ & $25 \mathrm{Nm} / \mathrm{kg}$ & $65 \%$ & $0.005 \mathrm{~kg}^{4} / \mathrm{Nm}^{4}(\tau \Delta t)$ \\
Li et al. $^{17}$ & $0.004 \mathrm{~J} / \mathrm{g}(4 \mathrm{Nm} / \mathrm{kg})$ & $\mathrm{N} / \mathrm{A}$ & $1.0\left(\mathrm{~g} / \mathrm{cm}^{3}\right)^{2}(\tau)$ \\
Mellar et al. $^{20}$ & $0.004 \mathrm{~J} / \mathrm{g}(4 \mathrm{Nm} / \mathrm{kg})$ & $10 \%$ & $1.0\left(\mathrm{~g} / \mathrm{cm}^{3}\right)^{2}(\tau)$ \\
\hline
\end{tabular}

*N/A-Not Available 